Pacific

Journal of

Mathematics

\title{
LEFSCHETZ FIBRATIONS WITH SMALL SLOPE
}

NAOYUKI MONDEN 


\title{
LEFSCHETZ FIBRATIONS WITH SMALL SLOPE
}

\author{
NAOYUKI MONDEN
}

\section{We construct Lefschetz fibrations over $S^{2}$ which do not satisfy the slope inequality. This disproves a conjecture of Hain.}

\section{Introduction}

Lefschetz fibrations have been an active area of research ever since the remarkable work in [Donaldson 1999] and [Gompf and Stipsicz 1999] revealed a close connection between them and symplectic 4-manifolds. In this paper, we consider the geography problem of Lefschetz fibrations over $S^{2}$, which derives from that of complex surfaces fibred over curves.

We are interested in two kinds of geography problems. Let $\sigma$ and $e$ be the signature and the Euler characteristic of a closed oriented smooth 4-manifold $X$, respectively. For an almost complex closed 4-manifold $X$, we set $K^{2}:=3 \sigma+2 e$ and $\chi_{h}:=(\sigma+e) / 4$ (the holomorphic Euler characteristic).

One is the geography problem for complex surfaces: the characterization of pairs $\left(K^{2}, \chi_{h}\right)$ corresponding to minimal complex surfaces. It is well known that any minimal complex surface of general type satisfies $K^{2}>0, \chi_{h}>0$, the Noether inequality $2 \chi_{h}-6 \leq K^{2}$ and the Bogomolov-Miyaoka-Yau inequality $K^{2} \leq 9 \chi_{h}$ (see [Barth et al. 1984], for example). The above geography problem can be extended to the symplectic 4-manifolds. However, Fintushel and Stern [1998] constructed Lefschetz fibration which does not satisfy the Noether inequality. In particular, for most pairs $(p, q)$ satisfying $p<2 q-6$, there exists a minimal symplectic 4-manifold with $p=K^{2}$ and $q=\chi_{h}$ (see [Gompf and Stipsicz 1999]). On the other hand, no examples of a minimal symplectic 4-manifold with $K^{2}>9 \chi_{h}$ have been found yet.

The other is the geography problem for complex surfaces fibred over curves. Hereafter, we assume $g \geq 2$. Let $f: S \rightarrow C$ be a relatively minimal holomorphic genus- $g$ fibration, where $S$ is a complex surface and $C$ is a complex curve of genus $k$. We define relative numerical invariants $\chi_{f}:=\chi_{h}-(g-1)(k-1)$ and $K_{f}^{2}:=K^{2}-8(g-1)(k-1)$ for $f: S \rightarrow C$. Then, we have two inequalities $\chi_{f} \geq 0$

MSC2010: primary 57R20; secondary 57N13, 14D06, 20F38.

Keywords: Lefschetz fibration, mapping class group, slope. 
and $K_{f}^{2} \geq 0$, known as Beauville's inequality (see [Beauville 1979]) and Arakelov's inequality (see [Arakelov 1971]), respectively. For $\chi_{f} \neq 0$, which is equivalent to the fact that $f$ is not a holomorphic bundle, we define $\lambda_{f}$ to be the quotient $K_{f}^{2} / \chi_{f}$, called the slope of $f$. Xiao [1987] proved that $4-4 / g \leq \lambda_{f} \leq 12$ (that is, $\left.(4-4 / g) \chi_{f} \leq K_{f}^{2} \leq 12 \chi_{f}\right)$. The former inequality is called the slope inequality. For a relatively minimal genus- $g$ Lefschetz fibration, $\chi_{f}, K_{f}^{2}$ and the slope $\lambda_{f}$ are defined in the same way as for complex surfaces fibred over curves. To the author's knowledge, the slope of all known Lefschetz fibrations over $S^{2}$ is greater than or equal to $4-4 / g$.

Conjecture 1.1 (Hain; see [Amorós et al. 2000, Question 5.10; Endo and Nagami 2005, Conjecture 4.12]). For every relatively minimal genus- $g$ Lefschetz fibration $f: X \rightarrow S^{2}$, the slope inequality $\lambda_{f} \geq 4-4 / g$ holds.

In this paper, we give a negative answer to Conjecture 1.1.

Theorem 3.1. For each $g \geq 3$, there exists a genus- $g$ Lefschetz fibration over $S^{2}$ with slope $\lambda_{f}=4-4 / g-1 / 3 g$ whose total space is simply connected.

Moreover, by fiber sum operations, we have the following results:

Corollary 3.6. For each $g \geq 3, m \geq 0$ and $l \geq 0$, there exists a genus- $g$ Lefschetz fibration $f_{m, l}: X_{m, l} \rightarrow S^{2}$ with slope $\lambda_{f_{m, l}}=4-4 / g-1 /(m+3) g$ such that $\pi_{1}\left(X_{m, l}\right)=1$. Moreover, if $(m, l) \neq(0,0)$, then $X_{m, l}$ is a minimal symplectic 4-manifold.

Corollary 3.7. For each $g \geq 3, m \geq 1$ and $l \geq 0$, there exists a genus- $g$ Lefschetz fibration $f_{m, l}^{\prime}: Y_{m, l} \rightarrow S^{2}$ with slope $\lambda_{f_{m, l}^{\prime}}=4-4 / g-1 / 2 g+1 /\left(2 \cdot 3^{m-1} g\right)$ such that $\pi_{1}\left(Y_{m, l}\right)=1$. Moreover, if $l \geq 1$, then $Y_{m, l}$ is a minimal symplectic 4-manifold.

As a consequence, we have the following results.

Corollary 4.2. The Lefschetz fibrations $f_{m, l}(m \geq 0)$ and $f_{m, l}^{\prime}(m \geq 2)$ are nonholomorphic.

Let $f: X \rightarrow S^{2}$ be a relatively minimal genus- $g$ Lefschetz fibration with $n>0$ singular fibers. From $e(X)=-4(g-1)+n$ and results from [Smith 1999; Stipsicz 1999; Ozbagci 2002], we have $\chi_{f}>0, K_{f}^{2} \geq 4 g-4$ and $\lambda_{f} \leq 10$. Moreover, it is well known that any hyperelliptic Lefschetz fibration satisfies the slope inequality. This fact follows from the signature formula for genus- $g$ hyperelliptic Lefschetz fibrations obtained by Matsumoto [1983; 1996] for $g=1,2$ and Endo [2000] for $g \geq 3$. Therefore, genus-2 Lefschetz fibrations satisfy the slope inequality. In particular, if $f$ is a hyperelliptic Lefschetz fibration with only nonseparating vanishing cycles, then $\lambda_{f}$ is equal to $4-4 / g$. For Lefschetz fibrations with $b_{2}^{+}=1$, we prove the following result. 
Theorem 5.1. Let $g \geq 2$ and let $f: X \rightarrow S^{2}$ be a genus- $g$ Lefschetz fibration with $b_{2}^{+}(X)=1$.

(1) If $X$ is not diffeomorphic to the blow-up of a ruled surface, then

$$
\begin{array}{ll}
4-4 / g \leq \lambda_{f} \leq 8 & \text { for } b_{1}(X)=0, \\
4 \leq \lambda_{f} \leq 8 & \text { for } b_{1}(X)=2 .
\end{array}
$$

(2) If $X$ is diffeomorphic to the blow-up of an $S^{2}$-bundle over $\Sigma_{k}$, then

$$
4+4(k-1) /(g-k) \leq \lambda_{f} \leq 8,
$$

and the lower bound is sharp.

The study of the slope of holomorphic fibrations was mainly motivated by Severi's inequality, which states that if $S$ is a minimal surface of general type of maximal Albanese dimension, then $K^{2} \geq 4 \chi_{h}$. Equivalently, if $K^{2}<4 \chi_{h}$, then $S$ is a surface fibred over $C$ of genus $b_{1}(S) / 2$. Severi [1932] claimed it, but his proof was not correct (see [Catanese 1983]). The inequality was independently posed as a conjecture by Reid [1979] and by Catanese [1983]. Xiao [1987] proved the conjecture when $S$ is a surface fibred over a curve of positive genus. He showed that if $S$ admits a holomorphic genus- $g$ fibration $f$ over $C$ of positive genus $k$ with $K^{2}<4 \chi_{h}+4(g-1)(k-1)$ (that is, $\left.\lambda_{f}<4\right)$, then $k=b_{1}(S) / 2$. Konno [1996] proved it in the case of even surfaces. The conjecture was solved by Manetti [2003] when $S$ has ample canonical bundle. Pardini [2005] proved the conjecture completely by using the slope inequality for holomorphic fibrations over $\mathbb{C P}^{1}$.

In Section 2, we review some standard facts on Lefschetz fibrations. Our main results are proved in Section 3. We give Lefschetz fibrations which violate the slope inequality. Consequently, we obtain examples of nonholomorphic Lefschetz fibrations in Section 4. In the last section, we investigate the slopes of Lefschetz fibrations with $b_{2}^{+}=1$.

Remark 1.2. The slope inequality of Conjecture 1.1 can be reformulated in terms of the Deligne-Mumford compactified moduli space of stable curves of genus $g$, denoted by $\bar{M}_{g}$, as follows. For a relatively minimal genus- $g$ Lefschetz fibration $f: X \rightarrow S^{2}$ with $n$ singular fibers, we obtain a symplectic structure on $X$ such that for all $x \in S^{2}, f^{-1}(x)$ is a pseudoholomorphic curve. Since a 2-dimensional almost-complex structure is integrable, $f^{-1}(x)$ determines a point in $\bar{M}_{g}$. Thus, we obtain the moduli map $\phi_{f}: S^{2} \rightarrow \bar{M}_{g}$ which is defined by $\phi_{f}(x)=\left[f^{-1}(x)\right] \in \bar{M}_{g}$ for $x \in S^{2}$. We denote by $\mathscr{H}_{g}$ the Hodge bundle on $\bar{M}_{g}$ with fiber the determinant line $\wedge^{g} H^{0}\left(C ; K_{C}\right)$, where $C$ is the set of critical points of $f$. By Smith's signature formula [1999] and the slope inequality, we have the following inequality:

$$
(8 g+4)\left\langle c_{1}\left(\mathcal{H}_{g}\right),\left[\phi_{f}\left(S^{2}\right)\right]\right\rangle-g \cdot n \geq 0 .
$$




\section{Preliminaries}

In this section, we first recall the definition and basic properties of Lefschetz fibrations. More details can be found in [Gompf and Stipsicz 1999].

Let $\Sigma_{g}$ be a closed oriented surface of genus $g \geq 2$ and let $\Gamma_{g}$ be the mapping class group of $\Sigma_{g}$, which is the group of isotopy classes of orientation-preserving diffeomorphisms of $\Sigma_{g}$. We denote by $t_{c}$ the right-handed Dehn twist about a simple closed curve $c$ on an oriented surface. The notation $t_{c} t_{d}$ means that we first apply $t_{d}$ then $t_{c}$.

Definition 2.1. Let $X$ be a closed, oriented smooth 4-manifold. A smooth map $f: X \rightarrow S^{2}$ is a genus- $g$ Lefschetz fibration if it satisfies the following conditions:

(i) $f$ has finitely many critical values $b_{1}, \ldots, b_{n} \in S^{2}$, and $f$ is a smooth $\Sigma_{g}$ bundle over $S^{2}-\left\{b_{1}, \ldots, b_{n}\right\}$.

(ii) For each $i(i=1, \ldots, n)$, there exists a unique critical point $p_{i}$ in the singular fiber $f^{-1}\left(b_{i}\right)$ such that about each $p_{i}$ and $b_{i}$ there are local complex coordinate charts agreeing with the orientations of $X$ and $S^{2}$ on which $f$ is of the form $f\left(z_{1}, z_{2}\right)=z_{1}^{2}+z_{2}^{2}$.

(iii) $f$ is relatively minimal (no fiber contains a $(-1)$-sphere).

Each singular fiber is obtained by collapsing a simple closed curve (the vanishing cycle) in the regular fiber. The monodromy of the fibration around a singular fiber is given by a right-handed Dehn twist along the corresponding vanishing cycle. A Lefschetz fibration $f: X \rightarrow S^{2}$ is holomorphic if there are complex structures on both $X$ and $S^{2}$ with holomorphic projection $f$.

Once we fix an identification of $\Sigma_{g}$ with the fiber over a base point of $S^{2}$, we can characterize the Lefschetz fibration $f: X \rightarrow S^{2}$ by its monodromy representation $\pi_{1}\left(S^{2}-\left\{b_{1}, \ldots, b_{n}\right\}\right) \rightarrow \Gamma_{g}$. This map is really an antihomomorphism, since elements of $\pi_{1}\left(S^{2}-\left\{b_{1}, \ldots, b_{n}\right\}\right)$ are written left-to-right and elements of $\Gamma_{g}$ are written right-to-left. Let $\gamma_{1}, \ldots, \gamma_{n}$ be an ordered system of generating loops for $\pi_{1}\left(S^{2}-\left\{b_{1}, \ldots, b_{n}\right\}\right)$, such that each $\gamma_{i}$ encircles only $b_{i}$ and $\prod \gamma_{i}$ is homotopically trivial. Thus, the monodromy of $f$ comprises a factorization

$$
t_{v_{n}} \ldots t_{v_{2}} t_{v_{1}}=1 \in \Gamma_{g},
$$

where $v_{i}$ are vanishing cycles of the singular fibers. This factorization is called the positive relator.

According to theorems of Kas [1980] and Matsumoto [1996], if $g \geq 2$, then the isomorphism class of a Lefschetz fibration is determined by a positive relator modulo simultaneous conjugations

$$
t_{v_{n}} \ldots t_{v_{2}} t_{v_{1}} \sim t_{\phi\left(v_{n}\right)} \ldots t_{\phi\left(v_{2}\right)} t_{\phi\left(v_{1}\right)} \quad \text { for all } \phi \in \Gamma_{g}
$$




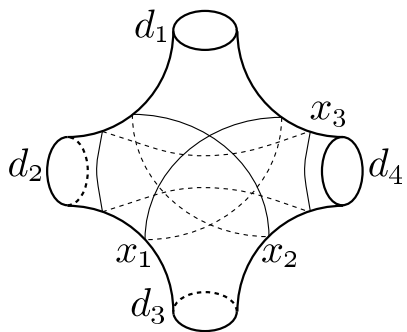

Figure 1. The curves $d_{1}, d_{2}, d_{3}, d_{4}, x_{1}, x_{2}, x_{3}$.

and elementary transformations

$$
\begin{aligned}
& t_{v_{n}} \ldots t_{v_{i+2}} t_{v_{i+1}} t_{v_{i}} t_{v_{i-1}} t_{v_{i-2}} \ldots t_{v_{1}} \sim t_{v_{n}} \ldots t_{v_{i+2}} t_{v_{i}} t_{t_{v_{i}}\left(v_{i+1}\right)} t_{v_{i-1}} t_{v_{i-2}} \ldots t_{v_{1}}, \\
& t_{v_{n}} \ldots t_{v_{i+2}} t_{v_{i+1}} t_{v_{i}} t_{v_{i-1}} t_{v_{i-2}} \ldots t_{v_{1}} \sim t_{v_{n}} \ldots t_{v_{i+2}} t_{v_{i+1}} t_{t_{v_{i}}}\left(v_{i-1}\right) \\
& t_{v_{i}} t_{v_{i-2}} \ldots t_{v_{1}} .
\end{aligned}
$$

Note that $\phi t_{v_{i}} \phi^{-1}=t_{\phi\left(v_{i}\right)}$. For all $\phi \in \Gamma_{g}$, let $\phi(\varrho)$ be the positive relator which is obtained by applying simultaneous conjugations by $\phi$ to a positive relator $\varrho$. We denote a Lefschetz fibration associated to a positive relator $\varrho \in \Gamma_{g}$ by $f_{\varrho}$ : $X_{\varrho} \rightarrow S^{2}$. Clearly, if $\varrho_{1} \sim \varrho_{2}$ in $\Gamma_{g}$ (that is, $\varrho_{2}$ is obtained by applying elementary transformations or simultaneous conjugations to $\varrho_{1}$ ), then

$$
\chi_{f_{\varrho_{1}}}=\chi_{f_{\varrho_{2}}} \quad \text { and } \quad K_{f_{\varrho_{1}}}^{2}=K_{{\ell_{2}}}^{2} \text {. }
$$

For positive relators $\varrho_{1}$ and $\varrho_{2}$ in $\Gamma_{g}$, the genus- $g$ Lefschetz fibration

$$
f_{\varrho_{1} \varrho_{2}}: X_{\varrho_{1} \varrho_{2}} \rightarrow S^{2}
$$

is the (trivial) fiber sum of $f_{\varrho_{1}}$ and $f_{\varrho_{2}}$. Since $\sigma\left(X_{\varrho_{1} \varrho_{2}}\right)=\sigma\left(X_{\varrho_{1}}\right)+\sigma\left(X_{\varrho_{2}}\right)$ and $e\left(X_{\varrho_{1} \varrho_{2}}\right)=e\left(X_{\varrho_{1}}\right)+e\left(X_{\varrho_{2}}\right)+4(g-1)$, we see that $\chi_{f_{\varrho_{1} \varrho_{2}}}=\chi_{f_{\varrho_{1}}}+\chi_{f_{\varrho_{2}}}$ and $K_{\varrho_{\varrho_{1} \varrho_{2}}}^{2}=K_{f_{\varrho_{1}}}^{2}+K_{f_{\varrho_{2}}}^{2}$. In particular, if $\varrho_{1} \sim \varrho_{2}$, then

$$
\chi_{f_{\varrho_{1} \varrho_{2}}}=2 \chi_{f_{\varrho_{1}}}=2 \chi_{f_{\varrho_{2}}} \quad \text { and } \quad K_{f_{\varrho_{1} \varrho_{2}}}^{2}=2 K_{f_{\varrho_{1}}}^{2}=2 K_{f_{\varrho_{2}}}^{2} .
$$

We next begin with a definition of the lantern relation (see [Dehn 1938; Johnson 1979]).

Definition 2.2. Let $\Sigma_{0}^{4}$ denote a sphere with 4 boundary components. Let $d_{1}, d_{2}$, $d_{3}, d_{4}$ be the 4 boundary curves of $\Sigma_{0}^{4}$ and let $x_{1}, x_{2}, x_{3}$ be the interior curves as shown in Figure 1. Then, we have the lantern relation

$$
t_{d_{1}} t_{d_{2}} t_{d_{3}} t_{d_{4}}=t_{x_{1}} t_{x_{2}} t_{x_{3}} \text {. }
$$

Let $\varrho$ be a positive relator of $\Gamma_{g}$. Let $d_{1}, d_{2}, d_{3}, d_{4}, x_{1}, x_{2}, x_{3}$ be curves as in Definition 2.2. Suppose that $\varrho$ includes $t_{d_{1}} t_{d_{2}} t_{d_{3}} t_{d_{4}}$ as a subword:

$$
\varrho=U \cdot t_{d_{1}} t_{d_{2}} t_{d_{3}} t_{d_{4}} \cdot V
$$


where $U$ and $V$ are products of right-handed Dehn twists. Then, by the lantern relation, the product of right-handed Dehn twists

$$
\varrho^{\prime}=U \cdot t_{x_{1}} t_{x_{2}} t_{x_{3}} \cdot V
$$

is also a positive relator of $\Gamma_{g}$.

This operation is one of substitution techniques introduced by Fuller.

Definition 2.3. We say that $\varrho^{\prime}$ is obtained by applying an $L$-substitution to $\varrho$. Conversely, $\varrho$ is said to be obtained by applying an $L^{-1}$-substitution to $\varrho^{\prime}$. We also call these two kinds of operations lantern substitutions.

Proposition 2.4 [Endo and Nagami 2005, Theorem 4.3 and Proposition 3.12]. Let $\varrho, \varrho^{\prime}$ be positive relators of $\Gamma_{g}$ and let $X_{\varrho}, X_{\varrho^{\prime}}$ be the corresponding Lefschetz fibrations over $S^{2}$, respectively. Suppose that $\varrho$ is obtained by applying an $L^{-1}$. substitution to $\varrho^{\prime}$. Then, $\sigma\left(X_{\varrho}\right)=\sigma\left(X_{\varrho^{\prime}}\right)-1$ and $e\left(X_{\varrho}\right)=e\left(X_{\varrho^{\prime}}\right)+1$. Therefore,

$$
\chi_{f_{\varrho}}=\chi_{f_{Q^{\prime}}} \quad \text { and } \quad K_{f_{\varrho}}^{2}=K_{f_{Q^{\prime}}}^{2}-1 .
$$

Remark 2.5. Endo and Gurtas [2010] showed that $X_{\varrho^{\prime}}$ is a rational blowdown of $X_{\varrho}$ introduced by Fintushel and Stern [1997]. Such relations were also generalized by Endo, Mark, and Van Horn-Morris [Endo et al. 2011].

\section{Main results}

In this section, we give a negative answer to Conjecture 1.1.

Theorem 3.1. For each $g \geq 3$, there exists a genus- $g$ Lefschetz fibration over $S^{2}$ with slope $\lambda_{f}=4-4 / g-1 / 3 g$ whose total space is simply connected.

In order to prove Theorem 3.1, we recall some standard facts on hyperelliptic Lefschetz fibrations. Let $\Delta_{g}$ be the hyperelliptic mapping class group of genus $g$, that is, the subgroup of $\Gamma_{g}$ which consists of all isotopy classes of orientationpreserving diffeomorphisms of $\Sigma_{g}$ commuting with the isotopy class of $\iota$, called the hyperelliptic involution. Note that $\Delta_{g}=\Gamma_{g}$ for $g=1,2$.

Definition 3.2. Let $\varrho=t_{a_{1}} \ldots t_{a_{n}}$ be a positive relator in $\Gamma_{g}$. A genus- $g$ Lefschetz fibration $f_{\varrho}: X_{\varrho} \rightarrow S^{2}$ is called hyperelliptic if for each $k \in\{1, \ldots, n\}, t_{a_{k}}$ is in $\Delta_{g}$. Equivalently, $\iota\left(a_{k}\right)=a_{k}$ for each $k$.

The following theorem was established in [Matsumoto 1983; 1996] for $g=1,2$ and in [Endo 2000] for $g \geq 3$. 

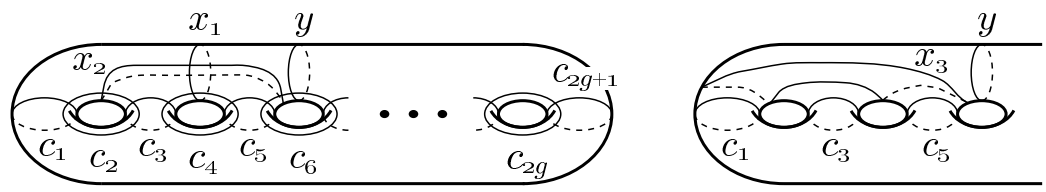

Figure 2. The curves $c_{1}, \ldots, c_{2 g+1}, x_{1}, x_{2}, x_{3}, y$.

Theorem 3.3 (Matsumoto, Endo). Let $f_{\varrho}: X_{\varrho} \rightarrow S^{2}$ be a genus- $g$ hyperelliptic Lefschetz fibration with $m$ nonseparating and

$$
s=\sum_{h=1}^{[g / 2]} s_{h}
$$

separating vanishing cycles, where $s_{h}$ denotes the number of separating vanishing cycles that separate $\Sigma_{g}$ into two surfaces, one of which has genus $h$. Then, we have

$$
\sigma\left(X_{\varrho}\right)=-\frac{g+1}{2 g+1} m+\sum_{h=1}^{[g / 2]}\left(\frac{4 h(g-h)}{2 g+1}-1\right) s_{h} .
$$

We need the following positive relator to prove Theorem 3.1. As shown in Figure 2, let $c_{1}, c_{2}, \ldots, c_{2 g+1}$ be the curves in $\Sigma_{g}$. We denote by $h_{g}\left(\in \Gamma_{g}\right)$ the product of $8 g+4$ right-handed Dehn twists

$$
h_{g}:=\left(t_{c_{1}} t_{c_{2}} \ldots t_{c_{2 g+1}}^{2} \ldots t_{c_{2}} t_{c_{1}}\right)^{2} .
$$

It is well known that $h_{g}$ is a positive relator in $\Delta_{g}$ and that $\sigma\left(X_{h_{g}}\right)=-4(g+1)$, by Theorem 3.3 and $e\left(X_{h_{g}}\right)=4(g+2)$. This gives $\chi_{f_{h g}}=g, K_{f_{h g}}^{2}=4 g-4$ and $\lambda_{f_{h g}}=4-4 / g$ (that is, $f_{h_{g}}$ is lying on the slope line).

Proof of Theorem 3.1. Suppose $g \geq 3$. Let $x_{1}, x_{2}, x_{3}, y$ be the curves as shown in Figure 2. Since $c_{1}, x_{i}$ are nonseparating curves, there exists a diffeomorphism $\phi_{i}$ such that $\phi_{i}\left(c_{1}\right)=x_{i}$. Hence, we have the following positive relator $r_{i}(i=1,2,3)$ :

$$
\begin{aligned}
r_{i}=\phi_{i} h_{g} \phi_{i}^{-1} & =\phi_{i}\left(t_{c_{1}} t_{c_{2}} \ldots t_{c_{2 g+1}}^{2} \ldots t_{c_{2}} t_{c_{1}}\right)^{2} \phi_{i}^{-1} \\
& =\left(t_{\phi_{i}\left(c_{1}\right)} t_{\phi_{i}\left(c_{2}\right)} \ldots t_{\phi_{i}\left(c_{2 g+1}\right)}^{2} \ldots t_{\phi_{i}\left(c_{2}\right)} t_{\phi_{i}\left(c_{1}\right)}\right)^{2} \\
& =\left(t_{x_{i}} t_{\phi_{i}\left(c_{2}\right)} \ldots t_{\phi_{i}\left(c_{2 g+1}\right)}^{2} \ldots t_{\phi_{i}\left(c_{2}\right)} t_{\phi_{i}\left(c_{1}\right)}\right)^{2} \\
& =1 \in \Gamma_{g} .
\end{aligned}
$$

Let $r_{g}^{\prime}=r_{1} r_{2} r_{3}=\left(t_{x_{1}} \ldots t_{\phi_{1}\left(c_{1}\right)}\right)^{2}\left(t_{x_{2}} \ldots\right)^{2}\left(t_{x_{3}} \ldots\right)^{2}$. Since $f_{r_{g}^{\prime}}$ is the fiber sum of $f_{r_{1}}, f_{r_{2}}$ and $f_{r_{3}}$ which are obtained by applying simultaneous conjugations to $h_{g}$, we have

$$
\chi_{f_{r_{g}^{\prime}}}=3 \chi_{f_{h_{g}}}=3 g \quad \text { and } \quad K_{f_{r_{g}^{\prime}}}^{2}=3 K_{f_{h_{g}}}^{2}=3(4 g-4)
$$


We apply elementary transformations to $r_{g}^{\prime}$ as follows:

$$
\begin{aligned}
& r_{g}^{\prime}=r_{1} r_{2} r_{3} \\
& =t_{x_{1}} t_{\phi_{1}\left(c_{2}\right)} \ldots t_{\phi_{1}\left(c_{2}\right)} t_{\phi_{1}\left(c_{1}\right)} \cdot t_{x_{2}} t_{\phi_{2}\left(c_{2}\right)} \ldots t_{\phi_{2}\left(c_{1}\right)} \cdot t_{x_{3}} t_{\phi_{3}\left(c_{2}\right)} \ldots t_{\phi_{3}\left(c_{1}\right)} \\
& \sim t_{x_{1}} t_{\phi_{1}\left(c_{2}\right)} \ldots t_{\phi_{1}\left(c_{2}\right)} t_{x_{2}} t_{x_{2}\left(\phi_{1}\left(c_{1}\right)\right)} t_{\phi_{2}\left(c_{2}\right)} \ldots t_{\phi_{2}\left(c_{1}\right)} \cdot t_{x_{3}} t_{\phi_{3}\left(c_{2}\right)} \ldots t_{\phi_{3}\left(c_{1}\right)} \\
& \text { : } \\
& \sim t_{x_{1}} t_{x_{2}} t_{t_{x_{2}}^{-1}\left(\phi_{1}\left(c_{2}\right)\right)} \ldots t_{t_{x_{2}}^{-1}\left(\phi_{1}\left(c_{2}\right)\right)} t_{t_{x_{2}}^{-1}\left(\phi_{1}\left(c_{1}\right)\right)} t_{\phi_{2}\left(c_{2}\right)} \ldots \underline{t_{\phi_{2}\left(c_{1}\right)} \cdot t_{x_{3}}} t_{\phi_{3}\left(c_{2}\right)} \ldots t_{\phi_{3}\left(c_{1}\right)} \\
& \sim t_{x_{1}} t_{x_{2}} t_{t_{x_{2}}\left(\phi_{1}\left(c_{2}\right)\right)} \ldots t_{t_{x_{2}}^{-1}\left(\phi_{1}\left(c_{2}\right)\right)} t_{t_{x_{2}}^{-1}\left(\phi_{1}\left(c_{1}\right)\right)} t_{\phi_{2}\left(c_{2}\right)} \ldots t_{x_{3}} t_{t_{x_{3}}^{-1}\left(\phi_{2}\left(c_{1}\right)\right)} t_{\phi_{3}\left(c_{2}\right)} \ldots t_{\phi_{3}\left(c_{1}\right)} \\
& \sim\left(t_{x_{1}} t_{x_{2}} t_{x_{3}}\right) W
\end{aligned}
$$

where $W$ is a product of $24 g+9$ right-handed Dehn twists. By the lantern relation, we get the following positive relator $r_{g}$ :

$$
r_{g}:=\left(t_{c_{1}} t_{c_{3}} t_{c_{5}} t_{y}\right) W .
$$

Since $r_{g}$ is obtained by applying an $L^{-1}$-substitution to $r_{g}^{\prime}$, by Proposition 2.4

$$
\chi_{f_{r g}}=3 g \quad \text { and } \quad K_{f_{r g}}^{2}=3(4 g-4)-1 .
$$

Then, the slope of $f_{r_{g}}$ is equal to $4-4 / g-1 / 3 g$.

Since it is easy to check that $r_{g}$ includes the Dehn twist about a curve $\phi_{3}\left(c_{i}\right)$ for $1 \leq i \leq 2 g+1, \pi_{1}\left(X_{r_{g}}\right)=1$. This follows from [Gompf and Stipsicz 1999] and the fact that $f_{r_{g}}$ has a section. This completes the proof of Theorem 3.1.

Remark 3.4. Since $r_{g}$ is obtained by applying an $L^{-1}$-substitution to $r_{g}^{\prime}, X_{r_{g}}$ is a rational blow-up of $X_{r_{g}^{\prime}}$. By applying elementary transformations to a relator corresponding to a Lefschetz fibration which is obtained by taking a twisted fiber sum with sufficiently many Lefschetz fibrations, we obtain a positive relator such that we can apply a monodromy substitution, which corresponds to the operation of rational blowdown (resp. rational blow-up) in [Endo et al. 2011], to it.

Remark 3.5. Miyachi and Shiga [2011] produced genus- $g$ Lefschetz fibrations over $\Sigma_{2 m}$ which do not satisfy the slope inequality.

Moreover, by fiber sum operations, we have the following results:

Corollary 3.6. For each $g \geq 3, m \geq 0$ and $l \geq 0$, there exists a genus- $g$ Lefschetz fibration $f_{m, l}: X_{m, l} \rightarrow S^{2}$ with slope $\lambda_{f_{m, l}}=4-4 / g-1 /(m+3) g$ such that $\pi_{1}\left(X_{m, l}\right)=1$. Moreover, if $(m, l) \neq(0,0)$, then $X_{m, l}$ is a minimal symplectic 4-manifold. 
Proof of Corollary 3.6. For any $m \geq 0$, we consider the Lefschetz fibration $f_{r_{g}} h_{g}^{m}$ : $X_{r_{g} h_{g}^{m}} \rightarrow S^{2}$ which is the fiber sum of $f_{r_{g}}$ and $m$ copies of $f_{h_{g}}$. Then,

$$
\begin{aligned}
& \chi_{f_{r_{g} h_{g}^{m}}}=\chi_{f_{r_{g}}}+m \chi_{f_{h_{g}}}=(3+m) g, \\
& K_{f_{r_{g} h_{g}^{m}}}^{2}=K_{f_{r_{g}}}^{2}+m K_{f_{h_{g}}}^{2}=(3+m)(4 g-4)-1 .
\end{aligned}
$$

Therefore, we obtain

$$
\lambda f_{r_{g} h_{g}^{m}}=4-4 / g-1 /(m+3) g .
$$

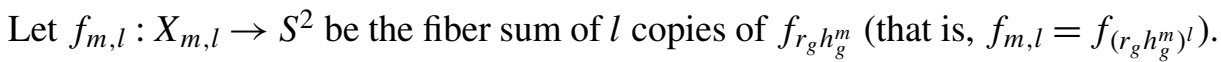
By Using (1) and an argument similar to the proof of Theorem 3.1, we have $\lambda_{f_{m, l}}=4-4 / g-1 /(m+3) g$ and $\pi_{1}\left(X_{m, l}\right)=1$. By the result of Usher [2006], $X_{m, l}$ is minimal for $(m, l) \neq(0,0)$. This completes the proof.

Corollary 3.7. For each $g \geq 3, m \geq 1$ and $l \geq 0$, there exists a genus- $g$ Lefschetz fibration $f_{m, l}^{\prime}: Y_{m, l} \rightarrow S^{2}$ with slope $\lambda_{f_{m, l}^{\prime}}=4-4 / g-1 / 2 g+1 /\left(2 \cdot 3^{m-1} g\right)$ such that $\pi_{1}\left(Y_{m, l}\right)=1$. Moreover, if $l \geq 1$, then $Y_{m, l}$ is a minimal symplectic 4-manifold.

Proof of Corollary 3.7. When we apply the argument of Theorem 3.1 again, with $\varrho_{1}=h_{g}$ replaced by $\varrho_{2}=r_{g}$, we obtain a genus- $g$ Lefschetz fibration $f_{\varrho_{3}}: X_{\varrho_{3}} \rightarrow S^{2}$ with

$$
\begin{aligned}
& \chi_{f_{\ell_{3}}}=3 \chi_{f_{\ell_{2}}}=3 \cdot 3 \chi_{f_{\ell_{1}}} \\
& K_{f_{e_{3}}}^{2}=3 K_{f_{\ell_{2}}}^{2}-1=3\left(3 K_{f_{\ell_{1}}}^{2}-1\right)-1 .
\end{aligned}
$$

By repeating this argument, we get a genus- $g$ Lefschetz fibration $f_{\varrho_{m}}(m \geq 1)$ with

$$
\begin{aligned}
\chi_{f_{\ell_{m}}} & =3^{m-1} \chi_{f_{\ell_{1}}}=3^{m-1} g, \\
K_{f_{\ell_{m}}}^{2} & =3\left(\ldots\left(3\left(3 K_{f_{\ell_{1}}}^{2}-1\right)-1\right) \ldots\right)-1=3^{m-1} K_{f_{\ell_{1}}}^{2}-3^{m-2}-\cdots-3-1 \\
& =3^{m-1}(4 g-4)-\left(3^{m-1}-1\right) / 2 .
\end{aligned}
$$

Therefore, $\lambda_{f_{\varrho_{m}}}=4-4 / g-1 / 2 g+1 /\left(2 \cdot 3^{m-1} g\right)$.

Let $f_{m, l}^{\prime}: Y_{m, l} \rightarrow S^{2}$ be the fiber sum of $l$ copies of $f_{\varrho_{m}}$, and so $\lambda_{f_{m, l}^{\prime}}=4-4 / g-$ $1 / 2 g+1 /\left(2 \cdot 3^{m-1} g\right)$. Similar to the proof of Corollary 3.6, we see that $\pi_{1}\left(Y_{m, l}\right)=1$ and that $Y_{m, l}$ is minimal for $l \geq 1$.

\section{Nonholomorphic Lefschetz fibrations}

There are various kinds of nonholomorphic Lefschetz fibrations. By fiber summing two copies of genus-2 Lefschetz fibration due to Matsumoto [1996], Ozbagci and Stipsicz [2000] constructed nonholomorphic genus-2 Lefschetz fibrations whose total space does not admit a complex structure. Korkmaz [2001] generalized their examples to $g \geq 3$. The above mentioned examples of Fintushel and Stern are 
also nonholomorphic Lefschetz fibrations. From the study of divisors in moduli space, Smith [2001] showed that a genus-3 Lefschetz fibration over $S^{2}$ which was produced by Fuller is nonholomorphic. Endo and Nagami [2005] constructed some examples of nonholomorphic Lefschetz fibrations which violate lower bounds of the slope for nonhyperelliptic fibrations of genus 3, 4 and 5 from the results of Konno [1991; 1993] and Chen [1993]. Hirose [2010] also gave some examples of $g=3,4$. In this section, we give new examples of nonholomorphic Lefschetz fibrations.

From the slope inequality for holomorphic fibrations, we have the following necessary condition for a Lefschetz fibration to be holomorphic:

Proposition 4.1 [Xiao 1987]. If a Lefschetz fibration $f$ is holomorphic, then the slope inequality $\lambda_{f} \geq 4-4 / g$ holds.

As a consequence, we have the following results.

Corollary 4.2. The Lefschetz fibrations $f_{m, l}(m \geq 0)$ and $f_{m, l}^{\prime}(m \geq 2)$ are nonholomorphic.

Remark 4.3. The above mentioned examples of Fintushel and Stern satisfy the slope inequality but violate the Noether inequality. On the other hand, $f_{m, l}$ and $f_{m, l}^{\prime}$ satisfy the Noether inequality but violate the slope inequality. Therefore, these two necessary conditions for a Lefschetz fibration to be holomorphic are independent in the sense that neither one implies the other.

\section{The slopes of Lefschetz fibrations with $b_{2}^{+}=1$}

We have the following natural question: Which Lefschetz fibrations satisfy the slope inequality? By Proposition 4.1, holomorphic Lefschetz fibrations satisfy the slope inequality. If a Lefschetz fibration is hyperelliptic, then $\lambda_{f} \geq 4-4 / g$. This fact can proved as follows. In the notation of Theorem 3.3, we have $e\left(X_{\varrho}\right)=$ $-4(g-1)+(m+s)$. Then, since $h \in\{1, \ldots,[g / 2]\}$ and $g \geq 2$, we have

$$
K_{f_{\varrho}}^{2}-(4-4 / g) \chi_{f_{\varrho}}=\sum_{h=1}^{[g / 2]} \frac{4 h(g-h)-g}{g} s_{h} \geq 0 .
$$

In particular, this means that for any hyperelliptic Lefschetz fibrations with only nonseparating vanishing cycles, $\lambda_{f}=4-4 / g$.

In this section, we investigate the slopes of Lefschetz fibrations with $b_{2}^{+}=1$. By combining the results of [Stipsicz 1999; 2002] and [Li 2000], we can show that Lefschetz fibrations with $b_{2}^{+}=1$ satisfy the slope inequality. Stipsicz showed that if $X \rightarrow S^{2}$ is a genus- $g$ Lefschetz fibration over $S^{2}$ with $b_{2}^{+}(X)=1$ and $X$ is not diffeomorphic to the blow-up of a ruled surface (that is, diffeomorphic to an $S^{2}$-bundle over $\Sigma_{k}$ ), then $b_{1}(X) \in\{0,2\}$ and $e \geq 0$ (see [Stipsicz 1999, Corollary 3.3 
and 3.5]). In particular, if $X$ is the blow-up of an $S^{2}$-bundle over $\Sigma_{k}$, then $k \leq g / 2$ (see [Li 2000, Proposition 4.4]). Then, we obtain the following result.

Theorem 5.1. Let $g \geq 2$ and let $f: X \rightarrow S^{2}$ be a genus- $g$ Lefschetz fibration with $b_{2}^{+}(X)=1$.

(1) If $X$ is not diffeomorphic to the blow-up of a ruled surface, then

$$
\begin{array}{ll}
4-4 / g \leq \lambda_{f} \leq 8 & \text { for } b_{1}(X)=0, \\
4 \leq \lambda_{f} \leq 8 & \text { for } b_{1}(X)=2 .
\end{array}
$$

(2) If $X$ is diffeomorphic to the blow-up of an $S^{2}$-bundle over $\Sigma_{k}$, then

$$
4+4(k-1) /(g-k) \leq \lambda_{f} \leq 8,
$$

and the lower bound is sharp.

An improvement of the previous result was suggested by the referee.

Proof of Theorem 5.1. For a genus- $g$ Lefschetz fibration, a regular fiber has zero self-intersection. Since the intersection form is nondegenerate, it follows that $b_{2}^{ \pm} \geq 1$. Let $f: X \rightarrow S^{2}$ be a nontrivial genus- $g$ Lefschetz fibration with $b_{2}^{+}(X)=1$. Note that $-4(g-1) \leq K^{2}$, and so $4(g-1) \leq K_{f}^{2}$ (see [Stipsicz 1999, Lemma 3.2]). Suppose that $X$ is not diffeomorphic to the blow-up of a ruled surface.

First, suppose that $b_{1}=0$. Since $b_{2}^{+}=1$ and $\chi_{f}=(\sigma+e) / 4+(g-1)=$ $\left(b_{2}^{+}-b_{1}+1\right) / 2+(g-1)=g$, we have $4(g-1) / g \leq K_{f}^{2} / \chi_{f}=\lambda_{f}$. On the other hand, since $K^{2}=3 \sigma+2 e=5 b_{2}^{+}-b_{2}^{-}+4-4 b_{1}=9-b_{2}^{-}$, by $b_{2}^{-} \geq 1$, we have $\lambda_{f}=K_{f}^{2} / \chi_{f}=\left\{9-b_{2}^{-}+8(g-1)\right\} / g \leq 8$.

Next, suppose $b_{1}=2$. Then, $\chi_{f}=g-1$. Therefore, by $4(g-1) \leq K_{f}^{2}$, we have $4 \leq \lambda_{f}$. Since $0 \leq e=2-2 b_{1}+b_{2}^{+}+b_{2}^{-}=2-4+1+b_{2}^{-}=-1+b_{2}^{-}$, we obtain $\lambda_{f}=\left\{1-b_{2}^{-}+8(g-1)\right\} /(g-1) \leq 8$.

Finally, suppose that $X$ is the $m$-fold blow-up of an $S^{2}$-bundle over $\Sigma_{k}$. Let $Y$ be the $S^{2}$-bundle over $\Sigma_{k}$. Then, since $b_{1}(Y)=2 k, b_{2}^{ \pm}(Y)=1$ and $X=Y \# m \overline{\mathbb{C P} \mathbb{P}^{2}}$, we have $b_{1}(X)=2 k, b_{2}^{+}(X)=1, b_{2}^{-}(X)=m+1, e(X)=4-4 k+m$ and $\sigma(X)=-m$. Hence, we have $\lambda_{f}=8-m /(g-k)$. From $m \geq 0, \lambda_{f} \leq 8$. We will give lower bounds for $\lambda_{f}$. By Lemma 3.2 in [Stipsicz 2002], 4(2k-g) $+m \leq 4$. We have $\lambda_{f} \geq 4+4(k-1) /(g-k)$ from $\lambda_{f}=8-m /(g-k), 4(2 k-g)+m \leq 4$ and $0 \leq k \leq g / 2$ [Li 2000, Proposition 4.4]. Fintushel and Stern [2004] showed that $\left(\Sigma_{k} \times S^{2}\right) \# 4 m \overline{\mathbb{C P}^{2}}$ admits a genus- $(2 k+m-1)$ Lefschetz fibration $f_{F S}$ over $S^{2}$. When $m=g-2 k+1$, we find $b_{2}^{+}=1$ and that $\lambda_{f_{F S}}=4+4(k-1) /(g-k)$.

Remark 5.2. If two Lefschetz fibrations $f_{1}$ and $f_{2}$ satisfy $\lambda_{f_{1}}, \lambda_{f_{2}} \geq 4-4 / g$, then the (twisted) fiber sum $f_{3}$ of $f_{1}$ and $f_{2}$ satisfies $\lambda_{f_{3}} \geq 4-4 / g$. 


\section{Acknowledgments}

The author would like to thank Hisaaki Endo for pointing out that the author's idea is effective in constructing a Lefschetz fibration which violates the slope inequality and for his encouragement. The author would like to express his gratitude to Kazuhiro Konno for his kind explanation of the history of the slope, and Mustafa Korkmaz and Kouichi Yasui for their comments on this paper. Finally, the referee's comments were especially helpful for improving the exposition.

\section{References}

[Amorós et al. 2000] J. Amorós, F. Bogomolov, L. Katzarkov, and T. Pantev, "Symplectic Lefschetz fibrations with arbitrary fundamental groups", J. Differential Geom. 54:3 (2000), 489-545. MR 2002g:57051 Zbl 1031.57021

[Arakelov 1971] S. J. Arakelov, "Families of algebraic curves with fixed degeneracies", Izv. Akad. Nauk SSSR Ser. Mat. 35 (1971), 1269-1293. In Russian; translated in Math. USSR Izv. 5 (1971), 1277-1302. MR 48 \#298 Zbl 0238.14012

[Barth et al. 1984] W. Barth, C. Peters, and A. Van de Ven, Compact complex surfaces, Ergebnisse der Mathematik und ihrer Grenzgebiete (3) 4, Springer, Berlin, 1984. MR 86c:32026 Zbl 0718.14023

[Beauville 1979] A. Beauville, "L'application canonique pour les surfaces de type général", Invent. Math. 55:2 (1979), 121-140. MR 81m:14025 Zbl 0403.14006

[Catanese 1983] F. Catanese, "Moduli of surfaces of general type", pp. 90-112 in Algebraic geometry: open problems (Ravello, 1982), edited by C. Ciliberto et al., Lecture Notes in Math. 997, Springer, Berlin, 1983. MR 84k:14026 Zbl 0517.14011

[Chen 1993] Z. J. Chen, "On the lower bound of the slope of a nonhyperelliptic fibration of genus 4", Internat. J. Math. 4:3 (1993), 367-378. MR 94g:14012 Zbl 0816.14006

[Dehn 1938] M. Dehn, “Die Gruppe der Abbildungsklassen”, Acta Math. 69:1 (1938), 135-206. MR 1555438 Zbl 0019.25301

[Donaldson 1999] S. K. Donaldson, "Lefschetz pencils on symplectic manifolds", J. Differential Geom. 53:2 (1999), 205-236. MR 2002g:53154 Zbl 1040.53094

[Endo 2000] H. Endo, "Meyer's signature cocycle and hyperelliptic fibrations", Math. Ann. 316:2 (2000), 237-257. MR 2001b:57047 Zbl 0948.57013

[Endo and Gurtas 2010] H. Endo and Y. Z. Gurtas, "Lantern relations and rational blowdowns", Proc. Amer. Math. Soc. 138:3 (2010), 1131-1142. MR 2011a:57047 Zbl 1198.57017

[Endo and Nagami 2005] H. Endo and S. Nagami, "Signature of relations in mapping class groups and non-holomorphic Lefschetz fibrations", Trans. Amer. Math. Soc. 357:8 (2005), 3179-3199. MR 2006g:57051 Zbl 1081.57023

[Endo et al. 2011] H. Endo, T. E. Mark, and J. Van Horn-Morris, "Monodromy substitutions and rational blowdowns", J. Topol. 4:1 (2011), 227-253. MR 2012b:57051 Zbl 1234.57037

[Fintushel and Stern 1997] R. Fintushel and R. J. Stern, "Rational blowdowns of smooth 4-manifolds", J. Differential Geom. 46:2 (1997), 181-235. MR 98j:57047 Zbl 0896.57022

[Fintushel and Stern 1998] R. Fintushel and R. J. Stern, "Constructions of smooth 4-manifolds", pp. 443-452 in Proceedings of the International Congress of Mathematicians, II (Berlin, 1998), edited by G. Fischer and U. Rehmann, 1998. MR 99g:57033 Zbl 0899.57012 
[Fintushel and Stern 2004] R. Fintushel and R. J. Stern, "Families of simply connected 4-manifolds with the same Seiberg-Witten invariants", Topology 43:6 (2004), 1449-1467. MR 2005d:57044 Zbl 1064.57036

[Gompf and Stipsicz 1999] R. E. Gompf and A. I. Stipsicz, 4-manifolds and Kirby calculus, Graduate Studies in Mathematics 20, Amer. Math. Soc., Providence, RI, 1999. MR 2000h:57038 Zbl 0933.57020

[Hirose 2010] S. Hirose, "Presentations of periodic maps on oriented closed surfaces of genera up to 4”, Osaka J. Math. 47:2 (2010), 385-421. MR 2011h:57025 Zbl 1202.57022

[Johnson 1979] D. L. Johnson, "Homeomorphisms of a surface which act trivially on homology", Proc. Amer. Math. Soc. 75:1 (1979), 119-125. MR 80h:57008 Zbl 0407.57003

[Kas 1980] A. Kas, "On the handlebody decomposition associated to a Lefschetz fibration", Pacific J. Math. 89:1 (1980), 89-104. MR 82f:57012 Zbl 0457.14011

[Konno 1991] K. Konno, "A note on surfaces with pencils of nonhyperelliptic curves of genus 3", Osaka J. Math. 28:3 (1991), 737-745. MR 93c:14035 Zbl 0766.14037

[Konno 1993] K. Konno, "Nonhyperelliptic fibrations of small genus and certain irregular canonical surfaces", Ann. Scuola Norm. Sup. Pisa Cl. Sci. (4) 20:4 (1993), 575-595. MR 95b:14026 Zbl 0822.14009

[Konno 1996] K. Konno, "Even canonical surfaces with small $K^{2}$, III", Nagoya Math. J. 143 (1996), 1-11. MR 98a:14051 Zbl 0872.14033

[Korkmaz 2001] M. Korkmaz, "Noncomplex smooth 4-manifolds with Lefschetz fibrations", Int. Math. Res. Not. 2001:3 (2001), 115-128. MR 2001m:57036 Zbl 0977.57020

[Li 2000] T.-J. Li, "Symplectic Parshin-Arakelov inequality", Int. Math. Res. Not. 2000:18 (2000), 941-954. MR 2001i:57038 Zbl 0961.57022

[Manetti 2003] M. Manetti, "Surfaces of Albanese general type and the Severi conjecture", Math. Nachr. 261-262:1 (2003), 105-122. MR 2004m:14081 Zbl 1044.14017

[Matsumoto 1983] Y. Matsumoto, "On 4-manifolds fibered by tori, II", Proc. Japan Acad. Ser. A Math. Sci. 59:3 (1983), 100-103. MR 84j:57010b Zbl 0532.55020

[Matsumoto 1996] Y. Matsumoto, "Lefschetz fibrations of genus two: a topological approach", pp. 123-148 in Topology and Teichmüller spaces (Katinkulta, 1995), edited by S. Kojima et al., World Scientific, 1996. MR 2000h:14038 Zbl 0921.57006

[Miyachi and Shiga 2011] H. Miyachi and H. Shiga, "Holonomies and the slope inequality of Lefschetz fibrations”, Proc. Amer. Math. Soc. 139:4 (2011), 1299-1307. MR 2012a:57036 Zbl 1226.14015

[Ozbagci 2002] B. Ozbagci, "Signatures of Lefschetz fibrations", Pacific J. Math. 202:1 (2002), 99-118. MR 2002k:57066 Zbl 1053.57013

[Ozbagci and Stipsicz 2000] B. Ozbagci and A. I. Stipsicz, "Noncomplex smooth 4-manifolds with genus-2 Lefschetz fibrations", Proc. Amer. Math. Soc. 128:10 (2000), 3125-3128. MR 2000m:57036 Zbl 0951.57015

[Pardini 2005] R. Pardini, "The Severi inequality $K^{2} \geq 4 \chi$ for surfaces of maximal Albanese dimension”, Invent. Math. 159:3 (2005), 669-672. MR 2006a:14065 Zbl 1082.14041

[Reid 1979] M. Reid, “ $\pi_{1}$ for surfaces with small $K^{2}$ ", pp. 534-544 in Algebraic geometry (Copenhagen, 1978), edited by K. Lønsted, Lecture Notes in Math. 732, Springer, Berlin, 1979. MR 81j: 14018 Zbl 0423.14021

[Severi 1932] F. Severi, "La serie canonica e la teoria delle serie principali di gruppi di punti sopra una superficie algebrica”, Comment. Math. Helv. 4:1 (1932), 268-326. MR 1509461 Zbl 0005.17602 
[Smith 1999] I. Smith, "Lefschetz fibrations and the Hodge bundle", Geom. Topol. 3 (1999), 211-233. MR 2000j:57059 Zbl 0929.53047

[Smith 2001] I. Smith, "Lefschetz pencils and divisors in moduli space", Geom. Topol. 5 (2001), 579-608. MR 2002f:57056 Zbl 1066.57030

[Stipsicz 1999] A. I. Stipsicz, "On the number of vanishing cycles in Lefschetz fibrations", Math. Res. Lett. 6:3-4 (1999), 449-456. MR 2000g:57046 Zbl 0955.57026

[Stipsicz 2002] A. I. Stipsicz, "Singular fibers in Lefschetz fibrations on manifolds with $b_{2}^{+}=1$ ", Topology Appl. 117:1 (2002), 9-21. MR 2002j:57048 Zbl 1007.53059

[Usher 2006] M. Usher, "Minimality and symplectic sums", Int. Math. Res. Not. 2006:16 (2006), Art. ID 49857. MR 2007h:53139 Zbl 1110.57017

[Xiao 1987] G. Xiao, "Fibered algebraic surfaces with low slope", Math. Ann. 276:3 (1987), 449-466. MR 88a:14046 Zbl 0596.14028

Received November 1, 2012. Revised May 19, 2013.

\author{
NAOYUKI MONDEN \\ DEPARTMENT OF MATHEMATICS \\ KYOTO UNIVERSITY \\ Graduate School of ScIENCE \\ KYOTO \\ JAPAN \\ n-monden@math.kyoto-u.ac.jp
}




\title{
PACIFIC JOURNAL OF MATHEMATICS
}

\author{
msp.org/pjm
}

Founded in 1951 by E. F. Beckenbach (1906-1982) and F. Wolf (1904-1989)

\section{EDITORS}

V. S. Varadarajan (Managing Editor)

Department of Mathematics

University of California

Los Angeles, CA 90095-1555

pacific@math.ucla.edu

Paul Balmer

Department of Mathematics

University of California

Los Angeles, CA 90095-1555

balmer@math.ucla.edu

Daryl Cooper

Department of Mathematics

University of California

Santa Barbara, CA 93106-3080 cooper@math.ucsb.edu

Jiang-Hua $\mathrm{Lu}$

Department of Mathematics

Pokfulam Rd., Hong Kong jhlu@maths.hku.hk
The University of Hong Kong

Don Blasius

Department of Mathematics University of California

Los Angeles, CA 90095-1555

blasius@math.ucla.edu

Robert Finn

Department of Mathematics Stanford University

Stanford, CA 94305-2125

finn@math.stanford.edu

Sorin Popa

Department of Mathematics

University of California

Los Angeles, CA 90095-1555 popa@math.ucla.edu

Paul Yang

Department of Mathematics Princeton University

Princeton NJ 08544-1000

yang@math.princeton.edu

\section{PRODUCTION}

Silvio Levy, Scientific Editor, production@msp.org

\section{SUPPORTING INSTITUTIONS}

ACADEMIA SINICA, TAIPEI

CALIFORNIA INST. OF TECHNOLOGY

INST. DE MATEMÁTICA PURA E APLICADA

KEIO UNIVERSITY

MATH. SCIENCES RESEARCH INSTITUTE

NEW MEXICO STATE UNIV.

OREGON STATE UNIV.

\author{
STANFORD UNIVERSITY \\ UNIV. OF BRITISH COLUMBIA \\ UNIV. OF CALIFORNIA, BERKELEY \\ UNIV. OF CALIFORNIA, DAVIS \\ UNIV. OF CALIFORNIA, LOS ANGELES \\ UNIV. OF CALIFORNIA, RIVERSIDE \\ UNIV. OF CALIFORNIA, SAN DIEGO \\ UNIV. OF CALIF., SANTA BARBARA
}

\author{
Vyjayanthi Chari \\ Department of Mathematics \\ University of California \\ Riverside, CA 92521-0135 \\ chari@math.ucr.edu \\ Kefeng Liu \\ Department of Mathematics \\ University of California \\ Los Angeles, CA 90095-1555 \\ liu@math.ucla.edu \\ Jie Qing \\ Department of Mathematics \\ University of California \\ Santa Cruz, CA 95064 \\ qing@cats.ucsc.edu
}

These supporting institutions contribute to the cost of publication of this Journal, but they are not owners or publishers and have no responsibility for its contents or policies.

See inside back cover or msp.org/pjm for submission instructions.

The subscription price for 2014 is US \$410/year for the electronic version, and \$535/year for print and electronic.

Subscriptions, requests for back issues and changes of subscribers address should be sent to Pacific Journal of Mathematics, P.O. Box 4163, Berkeley, CA 94704-0163, U.S.A. The Pacific Journal of Mathematics is indexed by Mathematical Reviews, Zentralblatt MATH, PASCAL CNRS Index, Referativnyi Zhurnal, Current Mathematical Publications and Web of Knowledge (Science Citation Index).

The Pacific Journal of Mathematics (ISSN 0030-8730) at the University of California, c/o Department of Mathematics, 798 Evans Hall \#3840, Berkeley, CA 94720-3840, is published twelve times a year. Periodical rate postage paid at Berkeley, CA 94704, and additional mailing offices. POSTMASTER: send address changes to Pacific Journal of Mathematics, P.O. Box 4163, Berkeley, CA 94704-0163.

PJM peer review and production are managed by EditFLOW ${ }^{\circledR}$ from Mathematical Sciences Publishers.

\section{PUBLISHED BY}

mathematical sciences publishers

nonprofit scientific publishing

http://msp.org/

(C) 2014 Mathematical Sciences Publishers 


\section{PACIFIC JOURNAL OF MATHEMATICS}

Volume $267 \quad$ No. $1 \quad$ January 2014

Numerical study of unbounded capillary surfaces

YASUNORI AOKI and HANS DE STERCK

Dual $R$-groups of the inner forms of $\operatorname{SL}(N)$

KUOK FAI CHAO and WEN-WEI LI

Automorphisms and quotients of quaternionic fake quadrics

AMIR DŽAmBIĆ and XAVIER ROULLEAU

Distance of bridge surfaces for links with essential meridional spheres 121

YEONHEE JANG

Normal states of type III factors

YASUYUKI KAWAHIGASHI, YOSHIKO OGATA and ERLING

STØRMER

Eigenvalues and entropies under the harmonic-Ricci flow

YI LI

Quantum extremal loop weight modules and monomial crystals

MATHIEU MANSUY

Lefschetz fibrations with small slope

NAOYUKI MONDEN 